\title{
The Missing Link in Star Cluster Evolution
}

\author{
Richard de Grijs, \\ Institute of Astronomy, University of Cambridge, Madingley Road, Cambridge CB3 OHA, \\ $U K$ \\ Nate Bastian, and Henny J.G.L.M. Lamers \\ Astronomical Institute, Utrecht University Princetonplein 5, 3584 CC Utrecht, The \\ Netherlands
}

\begin{abstract}
The currently most popular models for the dynamical evolution of star clusters predict that the power-law Cluster Luminosity Functions (CLFs) of young star cluster systems will be transformed rapidly into the universal Gaussian CLFs of old Milky Way-type "globular" cluster systems. Here, we provide the first evidence for a turn-over in the intermediate-age, approximately 1 Gyr-old CLF in the center of the nearby starburst galaxy M82, which very closely matches the universal CLFs of old Milky Way-type globular cluster systems. This provides an important test of both cluster disruption theories and hierarchical galaxy formation models. It also lends strong support to the scenario that these young cluster systems may eventually evolve into old Milky Way-type globular cluster systems. M82's proximity, its shortest known cluster disruption time-scale of any galaxy, and its well-defined peak of cluster formation make it an ideal candidate to probe the evolution of its star cluster system to fainter luminosities, and thus lower masses, than has been possible for any galaxy before.
\end{abstract}

Subject headings: galaxies: evolution - galaxies: individual (M82) - galaxies: starburst - galaxies: star clusters

\section{The Cluster Luminosity Function}

For old globular cluster systems, with ages in excess of $\sim 10^{10} \mathrm{yr}$, the shape of the cluster luminosity function (CLF) is well-established: it is roughly Gaussian, with an apparently universal peak luminosity, $M_{V}^{0} \simeq-7.4 \mathrm{mag}$, and a Gaussian FWHM $\sim 3$ mag (e.g., Whitmore et al. 1995, Harris 1996, 2001, Ashman \& Zepf 1998, Harris et al. 1998). On 
the other hand, the well-studied young, $\lesssim 2$ Gyr-old star cluster population in the Large Magellanic Cloud displays a power-law CLF of the form $N(L) \mathrm{d} L \propto L^{\alpha} \mathrm{d} L$, where $L$ is the cluster luminosity and $-2.0 \lesssim \alpha \lesssim-1.5$ (e.g., Elson \& Fall 1985, Elmegreen \& Efremov 1997).

Hubble Space Telescope (HST) observations are continuing to provide an increasing number of luminosity distributions for additional samples of young and intermediate-age compact star clusters in more distant galaxies (e.g., Whitmore \& Schweizer 1995, Schweizer et al. 1996, Miller et al. 1997, Zepf et al. 1999, de Grijs, O'Connell \& Gallagher 2001, Whitmore et al. 2002). Although a large number of studies have attempted to detect a turn-over in young or intermediate-age CLFs, the shapes of such young and intermediate-age CLFs have thus far been consistent with power laws down to the observational completeness thresholds.

Based on the observational evidence discussed above, the currently most popular globular cluster formation models suggest that the distribution of the initial cluster luminosities and, therefore, the corresponding distribution of their initial masses is closely approximated by a power law (Harris \& Pudritz 1994, McLaughlin \& Pudritz 1996, Elmegreen \& Efremov 1997, Ashman \& Zepf 2001) of the form $\mathrm{d} N(M) \mathrm{d} M \propto M^{\alpha} \mathrm{d} M$, where $-2.0 \lesssim \alpha \lesssim-1.5$. Several processes are proposed for the transformation of the CLF of young star cluster systems into the Gaussian or log-normal distributions characteristic of old globular cluster-type distributions. These include the preferential depletion of low-mass clusters both by evaporation due to two-body relaxation and by tidal interactions with the gravitational field of their host galaxy, and the preferential disruption of high-mass clusters by dynamical friction. However, the relative importance of these disruption processes is still controversial (see, e.g., Vesperini [2000, 2001], and Bromm \& Clarke [2002] for models that do not implicitly assume a power-law initial mass distribution). If the age range within a given cluster system is a significant fraction of the system's mean age, fading due to aging of the non-coeval cluster population also severely affects the shape of its CLF: the observed CLF will de dominated by the younger clusters, because a fraction of the older clusters in the system will have faded to below the observational detection limit, thus introducing further incompleteness effects.

The detection of a characteristic turn-over luminosity in young or intermediate-age CLFs would lend strong support to the popular (but thus far only speculative) scenario that the young star clusters observed in mergers are the analogues to the ubiquitous old globular clusters at younger ages. 


\section{The intermediate-age star cluster system in M82}

We now consider the intermediate-age star cluster system in the disturbed, late-type galaxy M82 in this context. M82, the nearest and best-studied starburst galaxy, has undergone multiple starburst episodes over the past $\sim 1-2$ Gyr. These were likely triggered by tidal interactions with M81 and/or other members of the M81/M82/NGC 3077 group (O’Connell \& Mangano 1978, Telesco 1988, Rieke et al. 1993, Yun, Ho \& Lo 1994, O’Connell et al. 1995, de Grijs et al. 2001, 2003).

In a recent study in which we focused on the fossil starburst site near the center of M82, region $\mathrm{B}$, we found a population of $\sim 110$ gravitationally bound evolved compact star clusters (de Grijs et al. 2001). Their properties appear to be consistent with the conclusion that they are evolved counterparts of the young compact star clusters detected in the galaxy's active core (O'Connell et al. 1995). We estimated ages for the M82 B cluster population from $\sim 30$ Myr to over 10 Gyr, with a peak at 1.0 Gyr (de Grijs et al. 2001, 2003), based on a comparison of broad-band optical and near-infrared HST colors with the Bruzual \& Charlot (2000; BC00) stellar evolutionary synthesis models.

These results suggest steady, continuing cluster formation in M82 B at a very modest rate at early times ( $>2$ Gyr ago) followed by a concentrated formation episode lasting from 500-1500 Myr ago and a subsequent suppression or decline of cluster formation (de Grijs et al. 2001, 2003, Parmentier, de Grijs \& Gilmore 2003). This finite burst of cluster formation makes the star cluster system in M82 B a good candidate to address the evolution of the CLF, because it provides a large sample of approximately coeval clusters (see Fig. 1a). Moreover, because of the proximity of M82, we have been able to probe the intermediate-age cluster population in M82 B to fainter absolute magnitudes, and thus lower masses, than has been possible before in other, more distant galaxies.

Figure 1a shows the star cluster formation rate in M82 B (de Grijs et al. 2003). We have highlighted the enhanced cluster formation episode, $8.4 \lesssim \log ($ Age $/$ yr $) \lesssim 9.4$. Uncertainties in the age determinations may have broadened the peak, so that the actual duration of the burst of cluster formation may have been shorter (de Grijs et al. 2001, 2003).

For the proper interpretation of the M82 B CLF, we need to correct the individual cluster luminosities for their range in ages (Meurer 1995, Fritze-v. Alvensleben 1999, de Grijs et al. 2001, 2003). Using the BC00 models, we have corrected the present-day absolute magnitudes of the clusters formed in the burst of cluster formation to those at a common, fiducial age of 1.0 Gyr. The results are shown in Fig. 1b for both the subsample of 42 clusters with well-defined ages (de Grijs et al. 2003), and for the full sample of 58 clusters

formed in the burst (open histogram). The corresponding mass distributions, obtained from 
the application of the BC00 mass-luminosity relation to the cluster luminosities, are shown in Fig. 1c. In both Figs. 1b and c, we have also indicated our conservative detection limit at $V=22.5 \mathrm{mag}$ (for which we are confident to have an almost fully complete sample; see de Grijs et al. [2001], their Fig. 7), or $M_{V}=-5.3 \mathrm{mag}$ (in case of no extinction), at an age of 1.0 Gyr, assuming a distance to M82 of $m-M=27.8$ (de Grijs et al. 2001). Since all sources in our sample are resolved, the sample is not contaminated by bright stars (see de Grijs et al. [2001] for a full discussion). We have shown that if we do not restrict ourselves to the limited age range of the burst, the age and mass distributions obtained for the subsample with well-determined ages and those for the full sample of M82 B clusters are internally fully consistent (de Grijs et al. 2003). However, if we impose age limits on our analysis in order to restrict our study to a coeval cluster population at $\sim 1 \mathrm{Gyr}$, the large uncertainties in the age determinations for the subset of the full sample with less well-determined ages lead us to conclude that the results for the full sample should be given less weight and be treated with caution. Those clusters that are not well fit (e.g., having less well-constrained ages and masses) are generally fainter and as such are artificialy skewed towards younger ages due to the well-known age-extinction degeneracy, which is more important for these clusters owing to their lower signal-to-noise ratios (see de Grijs et al. 2003 for more details).

In Fig. 2 we show the distribution of the M82 B clusters in the age vs. mass plane. The various (solid, dashed and dotted) lines overplotted on the figure show the expected effect of normal evolutionary fading of a synthetic single stellar population of an instantaneously formed cluster at our limiting magnitude. For ages $\leq 10^{9} \mathrm{yr}$, we show the unreddened fading line for various choices for the IMF, predicted by the Starburst99 (SB99) models (Leitherer et al. 1999). For older ages $\left(t \geq 10^{9} \mathrm{yr}\right)$, we show its extension predicted by the BC00 models. These predicted lower limits agree well with our data points, which therefore shows that we understand our selection effects to sufficient accuracy.

\section{Detection of a turn-over in an intermediate-age CLF}

Both the M82 B burst CLF and the corresponding mass distribution show a clear turnover at about 2 magnitudes brighter, and an order of magnitude more massive, than our detection limit, respectively. This is the first time that a turn-over has been detected for a coeval star cluster system as young as $\sim 1$ Gyr.

The characteristic turn-over mass of the M82 B clusters formed in the burst of cluster

formation, $M_{\mathrm{TO}} \simeq 1.2 \times 10^{5} M_{\odot}$ (de Grijs et al. 2003), is approximately half that of the old Galactic globular cluster system (Harris 1996). However, if we assume a power-law or Schechter-type initial cluster mass distribution in a Milky Way-type gravitational potential 
with strongly radially dependent radial anisotropy (Fall \& Zhang 2001), the peak of the mass distribution will change over time towards higher masses due to the effects of cluster disruption, which will preferentially deplete the lower-mass clusters. Furthermore, these models by Fall \& Zhang (2001) suggest that the turn-over of the cluster mass distribution will move towards higher masses by approximately $\Delta \log \left(M_{\mathrm{cl}} / M_{\odot}\right) \simeq+0.9$ by the time the cluster population reaches an age of $12 \mathrm{Gyr}$, similar to the median age of the Galactic globular cluster system. This implies that the star cluster system in M82 B will be dominated by higher masses than the Galactic globular cluster system when it reaches a similar age, and most of the present-day clusters will be depleted. This is most likely due to the fact that M82 $\mathrm{B}$ is characterized by the shortest known cluster disruption time-scale for any disk region of a galaxy, $\sim 30$ Myr for $10^{4} M_{\odot}$ clusters (de Grijs et al. 2003, using the method described in Boutloukos \& Lamers 2002).

This very short disruption time-scale suggests a significantly different gravitational potential, however, so that our estimate of the amount that the turn-over mass will move towards higher masses is, in fact, a lower limit. Additional support for this conclusion is given by the observation that the current turn-over mass is already $\Delta \log \left(M_{\mathrm{cl}} / M_{\odot}\right) \simeq+0.5$ more massive than the turn-over mass for a young star cluster system in a Galactic gravitational potential at a similar age, corresponding to $M_{\mathrm{TO} \text {,young }} \sim 7 \times 10^{4} M_{\odot}$ (Fall \& Zhang 2001). ${ }^{1}$ In addition, the width of the mass distribution in Fig. 1c is significantly smaller than that of the Galactic globular cluster mass function (Harris 1996), which again emphasizes the significant effects of the very short cluster disruption time-scale and the significantly different (and time-varying) gravitational potential governing the M82 system.

If the Fall \& Zhang (2001) models would apply to the M82 B cluster system, which we assume for the sake of the current discussion, the peak luminosity and width of the CLF will remain virtually unchanged for a Hubble time from its current age of $\sim 1$ Gyr (S.M. Fall, priv. comm.), due to the combination of evolutionary fading and continuing disruption processes (Whitmore et al. 2002). In that case, we can directly compare the intermediateage M82 B clusters to the old Galactic globular cluster population. The CLF of the M82 $\mathrm{B}$ cluster system is characterized by a turn-over luminosity of $M_{V}^{0}=-7.3 \pm 0.1 \mathrm{mag}$, and (within the Poissonian observational uncertainties) a Gaussian FWHM of $\sim 3.1$ mag. The M82 B CLF of clusters formed in the burst of cluster formation is therefore nearly identical

\footnotetext{
${ }^{1}$ We note, however, that a significant contributor to this apparent difference may be hidden in our choice for the IMF, which we have assumed to be roughly Salpeter-like. Despite a growing body of evidence for a universal IMF independent of the environment (see Gilmore [2001] for a review), significant departures from a Salpeter-type IMF (e.g., Smith \& Gallagher 2001) may give rise to larger-than-expected uncertainties in the derived mass distribution.
} 
to the Galactic globular CLF, although the old Galactic globular clusters are significantly more metal-poor than the roughly solar-abundance M82 B clusters (Parmentier et al. 2003). This difference in metallicity will, however, only affect the peak luminosity slightly, by less than 0.3 mag (Whitmore et al. 2002).

Finally, we note that the key ingredient of the Fall \& Zhang (2001) models is the radial anisotropy, which is a strong function of galactocentric distance. The net result of this assumption is that all of the Galactic globular clusters in their models have effectively the same pericenter. While this does not have to apply to the cluster system of M82 as a whole, we emphasize that the cluster system discussed in this letter is confined to a relatively small spatially confined region in the disk of M82, at the end of the central bar (cf. de Grijs 2001). These clusters have, therefore, very similar pericenters indeed.

\section{Summary and Implications}

Thus, here we have presented the first conclusive evidence for a clear turn-over in the CLF of a 1 Gyr-old, roughly coeval cluster population. The CLF shape and characteristic luminosity is nearly identical to that of the apparently universal CLFs of the old globular cluster systems in the Galaxy, M31, M87, and old elliptical galaxies (e.g., Whitmore et al. 1995, Harris 1996, 2001, Ashman \& Zepf 1998, Harris et al. 1998). This is likely to remain virtually unchanged for a Hubble time. We have also shown that with the very short characteristic cluster disruption time-scale governing M82 B, its cluster mass distribution will evolve towards a higher characteristic mass scale than for the Galactic globular clusters by the time it reaches a similar age. We argue, therefore, that this evidence, combined with the similar cluster sizes (de Grijs et al. 2001), lends strong support to a scenario in which the current M82 B cluster population will eventually evolve into a significantly depleted old Milky Way-type globular cluster system dominated by a small number of high-mass clusters. This implies that globular clusters, which were once thought to be the oldest building blocks of galaxies, are still forming today in galaxy interactions and mergers. However, they will likely be more metal-rich than the present-day old globular cluster systems.

This connection between young or intermediate-age star cluster systems and old globular clusters lends support to the hierarchical galaxy formation scenario. Old globular clusters were once thought to have been formed at the time of, or before, galaxy formation, i.e., during the first galaxy mergers. However, here we have shown that the evolved CLF of the compact star clusters in M82 B most likely to survive for a Hubble time will probably resemble the high-mass wing of the "universal" old globular cluster systems in the local Universe. Proto-globular cluster formation thus appears to be continuing until the present. 
In order to better constrain the future evolution of the M82 B star cluster system it is important to consider a range of models, characterized by fewer or perhaps different orbital restrictions (e.g., Baumgardt 1998, Vesperini 2000, 2001). This is, however, beyond the scope of the current letter and will be done in a subsequent paper (de Grijs et al., in prep.).

Acknowledgements - We acknowledge useful and interesting discussions with R.W. O'Connell and S.M. Fall. We also thank A. Helmi for a critical reading of the manuscript, and the anonymous referee for helpful suggestions. RdeG acknowledges support from the Particle Physics and Astronomy Research Council (PPARC) and from The British Council under the UK-Netherlands Partnership Programme in Science.

\section{REFERENCES}

Ashman, K.M. \& Zepf, S.E. 1998, Globular Cluster Systems, Cambridge University Press

Ashman, K.M. \& Zepf, S.E. 1998, AJ, 122, 1888

Baumgardt, H. 1998, A\&A, 330, 480

Boutloukos, S.G. \& Lamers, H.J.G.L.M. 2002, MNRAS, in press

Bromm, V. \& Clarke, C.J. 2002, ApJ, 566, L1

Bruzual, G. \& Charlot, S. 2000, updated version of Bruzual, G. \& Charlot, S. 1996, in: Leitherer, C., et al. PASP, 108, 996 (BC00)

de Grijs, R. 2001, A\&G, 42, 12

de Grijs, R., Bastian, N., \& Lamers, H.J.G.L.M. 2003, MNRAS, in press (astro-ph/0211420)

de Grijs, R., O'Connell, R.W., \& Gallagher, J.S. 2001, AJ, 121, 768

Elmegreen, B.G. \& Efremov, Y.N. 1997, ApJ, 480, 235

Elson, R.A.W. \& Fall, S.M. 1985, PASP, 97, 692

Fall, S.M. \& Zhang, Q. 2001, ApJ, 561, 751

Fritze-v. Alvensleben, U. 1999, A\&A, 342, L25

Gilmore, G. 2001, in Tacconi, L.J. \& Lutz, D., eds., Proc. Ringberg meeting, Starbursts: Near and Far. in press (astro-ph/0102189) 
Harris, W.E. 1996, AJ, 112, 1487

Harris, W.E. 2001, in: Star Clusters, Saas-Fee Advanced Course 28, Spinger-Verlag, 223

Harris, W.E., Harris, G.L.H., \& McLaughlin, D.E. 1998, AJ, 115, 1801

Harris, W.E. \& Pudritz, R.E. 1994, ApJ, 429, 177

Leitherer, C., Schaerer, D., Goldader, J.D., González Delgado, R.M., Robert, C., Kune, D.F., de Mello, D.F., Devost, D. \& Heckman, T.M. 1999, ApJS, 123, 3

McLaughlin, D.E. \& Pudritz, R.E. 1996, ApJ, 457, 578

Meurer, G.R. 1995, Nat, 375, 742

Miller, B.W., Whitmore, B.C., Schweizer, F., \& Fall, S.M. 1997, AJ, 114, 2381

O’Connell, R.W., Gallagher, J.S., Hunter, D.A. \& Colley, W.N. 1995, ApJ, 446, L1

O’Connell, R.W. \& Mangano, J.J. 1978, ApJ, 221, 62

Parmentier, G., de Grijs, R. \& Gilmore, G. 2003, MNRAS, submitted

Rieke, G.H., Loken, K., Rieke, M.J., \& Tamblyn, P. 1993, ApJ, 412, 99

Schweizer, F., Miller, B.W., Whitmore, B.C. \& Fall, S.M. 1996, AJ, 112, 1839

Smith, L.J. \& Gallagher, J.S. 2001, MNRAS, 326, 1027

Telesco, C.M. 1988, ARA\&A, 26, 343

Vesperini, E. 2001, MNRAS, 318, 841

Vesperini, E. 2001, MNRAS, 322, 247

Whitmore, B.C. \& Schweizer, F. 1995, AJ, 109, 960

Whitmore, B.C., Schweizer, F., Kundu, A., \& Miller, B.W. 2002, AJ, 124, 147

Whitmore, B.C., Sparks, W.B., Lucas, R.A., Macchetto, F.D., \& Biretta, J.A. 1995, ApJ, 454, L73

Yun, M.S., Ho, P.T.P. \& Lo, K.Y. 1994, Nat, 372, 530

Zepf, S.E., Ashman, K.M., English, J., Freeman, K.C., \& Sharples, R.M. 1999, AJ, 118, 752 

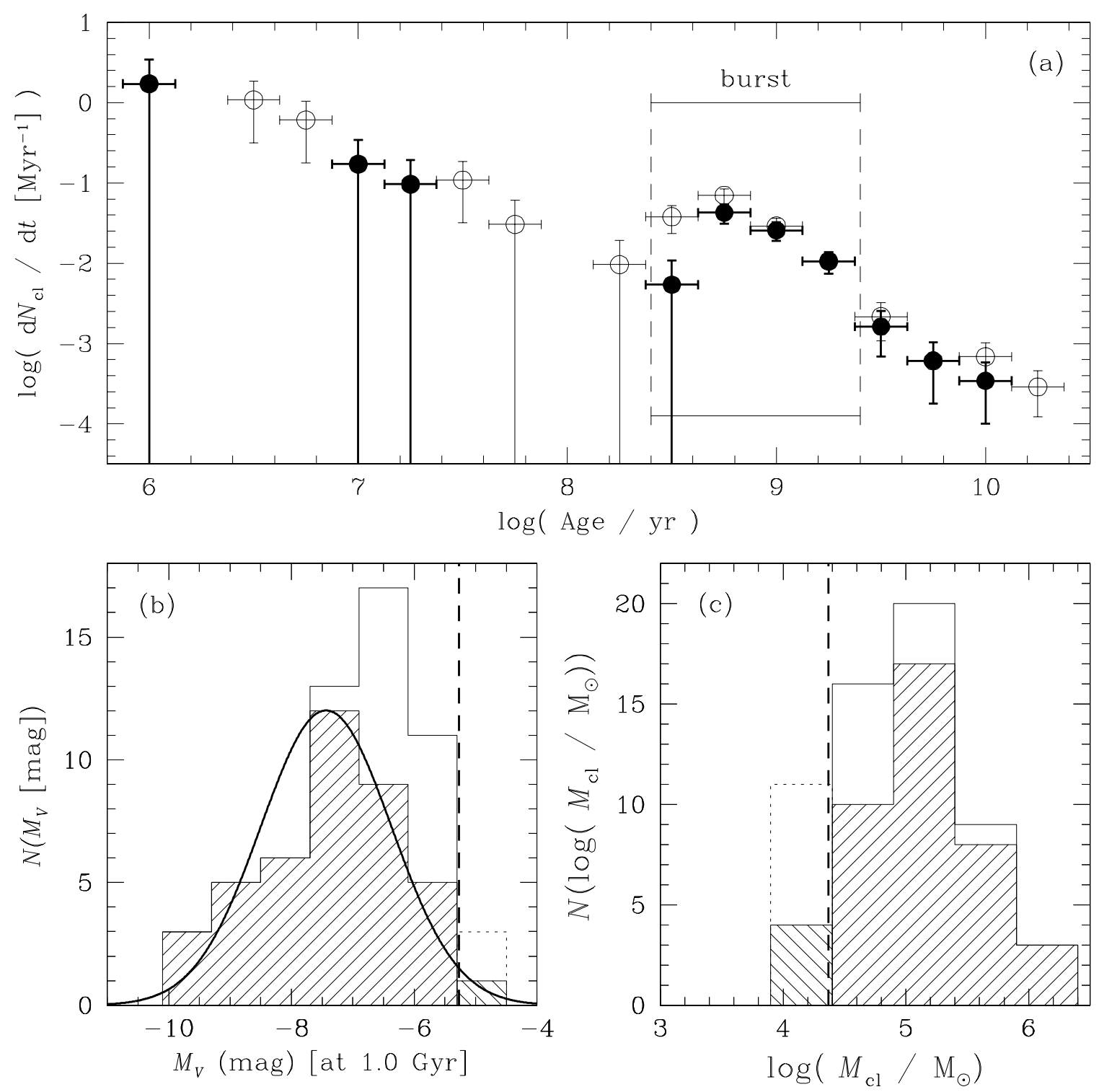

Fig. 1.- (a) The cluster formation rate (in number of clusters per Myr) as a function of age. Open circles: full sample; filled circles: clusters with well-determined ages (de Grijs et al. 2003). The age range dominated by the burst of cluster formation is indicated. (b) CLF of the clusters formed in the burst of cluster formation, $8.4 \leq \log$ (Age/yr) $\leq 9.4$. The shaded histograms correspond to the clusters with well-determined ages; the open histograms represent the entire cluster sample in this age range, as above. The Gaussian curve is the best fit to the shaded distribution. Finally, the vertical dashed line is our selection limit. (c) Mass distribution of the clusters formed in the burst of cluster formation; the coding is as in Fig. 1b. The expected effect of the $A_{V} \lesssim 0.2$ mag extinction (de Grijs et al. 2003) for the clusters with well-determined ages is a shift in mass towards higher masses of $\Delta \log \left(M_{\mathrm{cl}} / M_{\odot}\right) \lesssim 0.08$, which implies that the observed turnover is not a spurious effect due to varying extinction. 


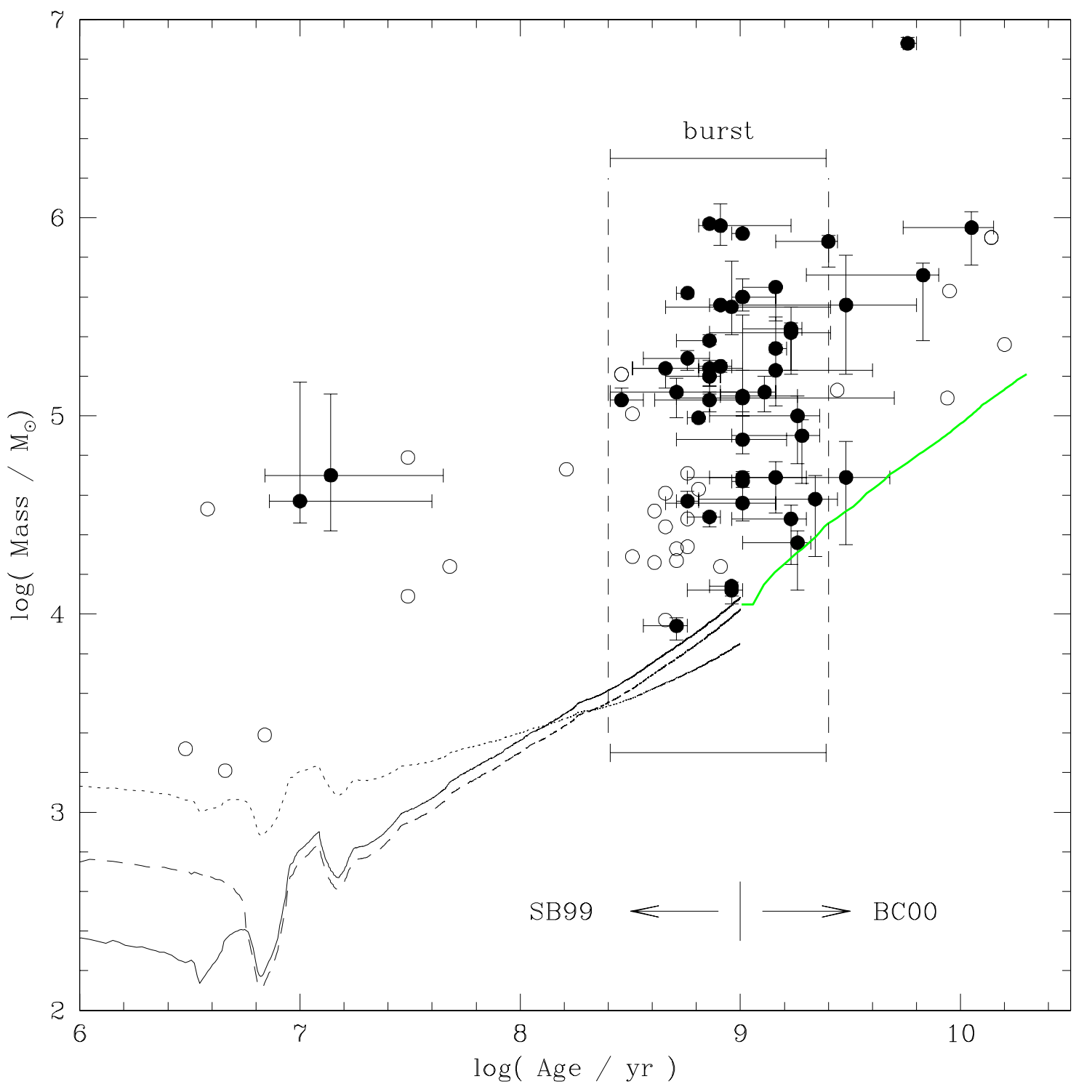

Fig. 2.- Distribution of the M82 B clusters in the (age vs. mass) plane. The age range dominated by the burst of cluster formation is indicated. The black dots represent clusters for which the total age range obtained is $(\log (\operatorname{Age}[\max ])-\log (\operatorname{Age}[\min ])) \leq 1.0$; the open circles are objects with more uncertain age determinations. Overplotted for ages up to 1.0 Gyr are the expected detection limits in the predicted by Starburst99 (SB99) for a range of IMFs (solid line: mass range $0.1-100 M_{\odot}$, IMF slope $\alpha=2.35$; dotted line: identical mass range, but $\alpha=3.30$; dashed line: mass range $0.1-30 M_{\odot}, \alpha=2.35$ ); for older ages, we use the BC00 models for a standard IMF (mass range $0.1-100 M_{\odot}, \alpha=2.35$ ). These model predictions are based on a very conservative detection limit of $V=22.5$ (see de Grijs et al. 2001) and $(m-M)_{\mathrm{M} 82}=27.8$, assuming no extinction. For a nominal extinction of $A_{V}=0.2 \mathrm{mag}$, expected for the clusters with well-determined ages (de Grijs et al. 2003), the detection limit is expected to shift to higher masses by $\Delta \log \left(M_{\mathrm{cl}} / M_{\odot}\right)=0.08$, which is well within the uncertainties associated with our mass determinations. 